\title{
Prediabetes Diagnosis and Treatment in Primary Care
}

\author{
Arch G. Mainous III, PhD, Rebecca J. Tanner, MA, and Richard Baker, MD
}

Background: The increasing prevalence of diabetes is a major health problem. The detection and treatment of prediabetes can delay the onset of diabetes and presents an important diabetes prevention strategy.

Methods: Using data from the 2012 National Ambulatory Medical Care Survey, we studied visits by adults aged $\geq 45$ years without diagnosed diabetes who had an $\mathrm{HbA}_{1 \mathrm{c}}$ test within 90 days of the visit ( $n=518$ unweighted visits; $n=11,167,004$ weighted visits). $\mathrm{HbA}_{1 \mathrm{c}}$ results were categorized into normal, prediabetes, and diabetes, and we examined patient characteristics (age, sex, race, payer type, body mass index) and treatment of prediabetes.

Results: Among visiting adults, $54.6 \%$ had a normal $\mathrm{HbA}_{1 \mathrm{c}}$ value, $33.6 \%$ had prediabetes, and $11.9 \%$ had diabetes. Of those patient visits with $\mathrm{HbA}_{1 \mathrm{c}}$ consistent with prediabetes, the number of patients diagnosed with prediabetes was too low for a reliable population estimate. Indication of treatment in the medical record (lifestyle modification counseling and/or metformin) was present in $23.0 \%$ of those with diagnosed or undiagnosed prediabetes. The most common treatment was lifestyle modification counseling.

Conclusions: Our findings show that there are missed opportunities for diabetes prevention in primary care. Providers need to change their approach to prediabetes and play a more effective role in preventing diabetes. (J Am Board Fam Med 2016;29:283-285.)

Keywords: Diabetes Mellitus, Prediabetes, Primary Health Care

Prediabetes is a high-risk state for developing diabetes. ${ }^{1}$ Recent data have shown that in developed countries more than a third of adults have prediabetes. $^{2,3}$ Detection of prediabetes is a fundamental strategy to keep people from transitioning to diabetes. ${ }^{1-5}$ Once detected, prediabetes should be acknowledged with a treatment plan (metformin or intense lifestyle intervention) to prevent or slow the transition to diabetes. ${ }^{6}$ However, it is unclear whether primary care physicians diagnose predia-

This article was externally peer reviewed.

Submitted 11 August 2015; revised 19 October 2015; accepted 28 October 2015.

From the Department of Health Services Research, Management and Policy, University of Florida, Gainesville (AGM, RJT); the Department of Health Sciences, University of Leicester, Leicester, UK (RB).

Funding: This study was funded in part by a grant from the Leicester City Council.

Conflict of interest: none declared.

Corresponding author: Arch G. Mainous III, PhD, Department of Health Services Research, Management and Policy, University of Florida, PO Box 100195, Gainesville, FL 32610-0195 (E-mail: arch.mainous@ufl.edu). betes and adjust the treatment plan in light of $\mathrm{HbA}_{1 \mathrm{c}}$ results.

\section{Methods}

We analyzed data from the 2012 National Ambulatory Medical Care Survey (NAMCS), a national probability sample survey of ambulatory medical care visits to office-based physicians that allows for national estimates regarding US medical care. ${ }^{7} \mathrm{We}$ focused on individuals ages 45 and older because that age group is consistent with the 2012 American Diabetes Association screening recommendations for prediabetes. ${ }^{1} \mathrm{We}$ included results of $\mathrm{HbA}_{1 \mathrm{c}}$ tests ordered within the 90 days preceding the sampled visit because that result would still be considered current for decision making at that visit. The sampled visits were to a general/family medicine or internal medicine provider. A total of 518 visits were available for analysis, and when these visits are weighted for the population and the analysis controlled for the complex sampling design, 
Table 1. Characteristics of Patients with No Diabetes Diagnosis and a Current $\mathrm{HbA}_{1 \mathrm{c}}$ Test, Aged 45 and older

\begin{tabular}{lr}
\hline Unweighted sample size, $\mathrm{n}$ & 518 \\
Weighted sample size, $\mathrm{n}$ & $1,167,004$ \\
Age (years), \% & 60.5 \\
$45-64$ & 39.5 \\
$\geq 65$ & \\
Sex, \% & 53.3 \\
Male & 46.7 \\
Female & \\
Race/ethnicity, \% & 66 \\
Non-Hispanic white & 6.4 \\
Non-Hispanic black & 18.8 \\
Hispanic & $8.7^{*}$ \\
Non-Hispanic other & \\
Payer type, \% & 47.6 \\
Private Insurance & 50.3 \\
Medicare/Medicaid/CHIP & $2.1^{*}$ \\
Other & \\
BMI category, \% & 13.7 \\
Underweight/healthy weight & 56 \\
Overweight & 30.3 \\
Obese & \\
HbA ${ }_{1 \mathrm{c}}$ category, \% & 54.6 \\
Normal (<5.7\%) & 33.6 \\
Prediabetes (5.7-6.4\%) & \\
Diabetes (>6.4\%) & \\
\hline
\end{tabular}

*Unreliable estimate.

CHIP, Children's Health Insurance Program.

they represent 1,167,004 office-based ambulatory medical care visits.

Prediabetes was defined as $\mathrm{HbA}_{1 \mathrm{c}}$ within the range of $5.7 \%$ to $6.4 \% .{ }^{1}$ Individuals were considered to have diagnosed prediabetes if 1 of the 3 diagnosis fields was the International Classification of Diseases, 9th Revision, code for prediabetes (790.29).

To account for the complex sampling design of the NAMCS, we used SAS software version 9.4 (SAS Institute, Cary, NC) for all analyses. We examined the characteristics of patients in both the full sample and the sample of patients with a positive test for prediabetes. We conducted logistic regression to examine the impact of $\mathrm{HbA}_{1 \mathrm{c}}$ level and other patient characteristics on receiving treatment for prediabetes among individuals with a current prediabetes test result and prediabetes. $\mathrm{HbA}_{1 \mathrm{c}}$ level was categorized as low prediabetes $(5.7 \%$ to $5.9 \%$ ) and high prediabetes (6.0\% to $6.4 \%)$. Listwise deletion was used in cases with missing data.
In analyzing NAMCS, the National Center for Health Statistics states that any frequencies with fewer than 30 respondents are considered unreliable and should not be used to make population estimates. Estimates that are considered unreliable are noted in the text when appropriate.

\section{Results}

Table 1 indicates characteristics of individuals with no previous diagnosis of diabetes and a current $\mathrm{HbA}_{1 \mathrm{c}}$ test. Rates were similar between men and women, with $40.0 \%$ of women and $36.5 \%$ of men having prediabetes. The most commonly occurring (in $16.3 \%$ ) primary diagnosis for patients with prediabetes was hypertensive disease (International Classification of Diseases, 9th Revision, codes 401 to 405). Although the results of the $\mathrm{HbA}_{1 \mathrm{c}}$ test were available to the participating physician, who recorded his or her actions in the NAMCS, the number of patients who received a diagnosis of prediabetes was too small to allow reliable estimates to be produced (the estimate was $0.92 \%$ ).

There were no significant differences in receiving treatments based on $\mathrm{HbA}_{1 \mathrm{c}}$ level. Of patients with an $\mathrm{HbA}_{1 \mathrm{c}}$ level between $5.7 \%$ and $5.9 \%$,

Table 2. Logistic Regression Results for Treatment of Prediabetes in Patients Who Had a Current $\mathrm{HbA}_{1 \mathrm{c}}$ Test and Prediabetes

Odds Ratio $(95 \% \mathrm{CI})$

Sex

Male 1.00

Female $1.53(0.51-4.57)$

Age (years)

$45-64$

1.00

$\geq 65$

$1.16(0.38-3.54)$

Insurance status

Private Insurance

Other

$0.53(0.16-1.74)$

1.00

Race/ethnicity

Non-Hispanic white

1.00

Nonwhite

$0.50(0.17-1.47)$

BMI category

Underweight/normal weight

1.00

Overweight

$0.31(0.09-1.05)$

Obese

$0.28(0.07-1.09)$

$\mathrm{HbA}_{1 \mathrm{c}}$

$5.7-5.9 \%$

1.00

$\geq 6.0 \%$

$0.80(0.32-2.00)$

BMI, body mass index; CI, confidence interval. 
$22.6 \%$ received treatment for prediabetes, compared with $23.5 \%$ of patients with an $\mathrm{HbA}_{1 \mathrm{c}}$ level between $6.0 \%$ and $6.4 \%(P=.91)$. Table 2 shows the results of the logistic regression model for receiving treatment. No variables included in the model were significant, including the $\mathrm{HbA}_{1 \mathrm{c}}$ test results.

\section{Discussion}

Considering the large proportion of patients with an $\mathrm{HbA}_{1 \mathrm{c}}$ test that is consistent with prediabetes, the number of patients diagnosed was too small to make a reliable population estimate, and three fourths of those with prediabetes were not provided with an appropriate treatment plan. This lack of diagnosis should be considered in light of the fact that the most common primary diagnosis for that visit was hypertension, a common comorbid condition with diabetes.

Our study does not explain why physicians did not record a prediabetes diagnosis and offer patients effective interventions to delay the onset of diabetes. Potential explanations include lack of awareness of the potential effect of interventions in reducing diabetes risk, lack of access to providers of dietary and exercise advice, and therapeutic inertia. There is also a possible concern among some clinicians that diagnosing prediabetes overmedicalizes a condition that is not a disease. ${ }^{8}$ For some patients with other morbidities, addressing prediabetes may not have been regarded as a priority. Further, it is possible that an artifact of the NAMCS data, which limits the physician to 3 diagnoses, may have created an undercount of diagnoses of prediabetes because there was not enough room to list it if it was considered the 4th or higher diagnosis. Finally, although we were evaluating ambulatory practice in the United States, the American Diabetes Association's $\mathrm{HbA}_{1 \mathrm{c}}$ range as a definition of prediabetes used in this study is not universally adopted around the world. ${ }^{9}$ As such, glucose tolerance testing may be an advisable further evaluation step to clarify the diagnostic status of individuals with $\mathrm{HbA}_{1 \mathrm{c}}$ of $5.7 \%$ to $6.4 \%$. Nevertheless, the finding that most patients with confirmed prediabetes do not receive appropriate care suggests that the approach of primary care toward prediabetes needs to change if we are to effectively prevent diabetes.

\section{References}

1. American Diabetes Association. Diagnosis and classification of diabetes mellitus. Diabetes Care. 2012; 35:S64-71.

2. Bullard KM, Saydah SH, Imperatore G, et al. Secular changes in U.S. prediabetes prevalence defined by hemoglobin A1c and fasting plasma glucose: $\mathrm{Na}-$ tional Health and Nutrition Examination Surveys, 1999-2010. Diabetes Care 2013;36:2286-93.

3. Mainous AG 3rd, Tanner RJ, Baker R, Zayas CE, Harle CA. Prevalence of prediabetes in England from 2003 to 2011: population-based, cross-sectional study. BMJ Open 2014;4:e005002.

4. Selph S, Dana T, Blazina I, Bougatsos C, Patel H, Chou R. Screening for type 2 diabetes mellitus: a systematic review for the U.S. Preventive Services Task Force. Ann Intern Med 2015;162:765-76.

5. U.S. Preventive Services Task Force. Final recommendation statement: abnormal blood glucose and type 2 diabetes mellitus: Screening. U.S. Preventive Services Task Force. December 2015. Available from: http://www.uspreventiveservicestaskforce.org/ Page/Document/RecommendationStatementFinal/ screening-for-abnormal-blood-glucose-and-type-2diabetes. Accessed January 19, 2016.

6. Dunkley AJ, Bodicoat DH, Greaves CJ, et al. Diabetes prevention in the real world: effectiveness of pragmatic lifestyle interventions for the prevention of type 2 diabetes and of the impact of adherence to guideline recommendations: a systematic review and meta-analysis. Diabetes Care 2014;37:922-33.

7. National Center for Health Statistics. Questionnaires, datasets, and related documentation. 2012 NAMCS. Atlanta: Centers for Disease Control and Prevention; 2015. Available from: ftp://ftp.cdc.gov/pub/Health_Statistics /NCHS/Dataset_Documentation/NAMCS/. Accessed October 19, 2015.

8. Yudkin JS, Montori VM. The epidemic of pre-diabetes: the medicine and the politics. BMJ 2014;349: g4485.

9. National Institute for Health and Care Excellence. Type 2 diabetes: prevention in people at high risk. Recommendation 1, risk assessment. NICE guidelines PH38. Published July 2012. Available from: http://www.nice.org.uk/guidance/ph38/chapter/ 1-Recommendations\#recommendation-1-riskassessment. Accessed October 19, 2015. 